\title{
CUIDADOS VETERINARIOS: ¿Atender aves, vacunos o mascotas?
}

\author{
José Luis Wakabayashi \\ UNIVERSIDAD ESAN \\ jwakabayashi@esan.edu.pe
}

\author{
Armando Borda \\ UNIVERSIDAD ESAN \\ aborda@esan.edu.pe
}

\begin{abstract}
Resumen
Empresa dedicada a importar y comercializar productos veterinarios debe gran parte de su crecimiento a la representación exclusiva de la marca Tasmil, líder mundial en productos destinados a mantener la salud animal. Si bien estos productos pueden dirigirse a los sectores avícola, ganadero, porcícola y de mascotas, en la actualidad las ventas de la empresa están concentradas (80\%) en el rubro avícola, el sector pecuario más organizado y con mayor potencial de desarrollo en el Perú. Ahora la empresa quiere seguir creciendo, pero en su línea tradicional ya llegó a su tope. Además, enfrenta restricciones de liquidez, a causa de la poca rotación de algunas líneas de productos dirigidos a otros sectores pecuarios. Finalmente, siempre se corre el riesgo de perder la representación exclusiva de Tasmil o, en el peor de los casos, de que esta encuentre atractivo el sector avícola y decida operar directamente en el país.
\end{abstract}

Palabras clave: manejo de clientes estratégicos, clientes rentables, clasificación de cartera, diversificación de producto-mercado, planeamiento estratégico.

\begin{abstract}
Company dedicated to the import and marketing of veterinary products, owes most of its growth to the exclusive representation of the Tasmil brand, a world leader in animal health products. While the products might be geared at the poultry, cattle, pig, and pet sectors, currently $80 \%$ of the company's sales are concentrated in the poultry market, which is the most organized livestock market in Peru, with the highest growth potential. The company wishes to continue growing, but it has already reached the limit in its traditional product line. Moreover, it faces liquidity restrictions due to the decreased turnover of some product lines aimed at the livestock sector. Finally, there is always the risk of loosing the Tasmil representation, or even worse, that Tasmil is attracted by the poultry market and decides to operate directly in the country.
\end{abstract}

Key words: strategic client management, profitable clients, portfolio classification, product-market diversification, strategic planning. 
A rturo Alva, fundador y presidente del directorio de Cuidados Veterinarios, revisaba la evolución del negocio y se sentía satisfecho. En los últimos diez años los volúmenes importados se habían incrementado en más de 15 veces, las ventas habían crecido en la misma proporción y las relaciones con sus principales clientes se consolidaban. La oportunidad de negocio detectada a inicios de los anõs noventa daba sus frutos: Cuidados Veterinarios ocupaba el puesto 920 entre las empresas más grandes del país ${ }^{1}$.

Sin embargo, el señor Alva tenía algunas dudas acerca de los sectores que atendía y se preguntaba si la tendencia registrada hasta el momento se mantendría o si había llegado la hora de hacer cambios para asegurar el crecimiento de la empresa. ¿Debían concentrarse en sus líneas actuales de producto o debían buscar nuevas líneas para desarrollarlas? ¿Debían incursionar en nuevas unidades estratégicas de negocio? ¿Debían concentrarse en desarrollar estrategias relacionales con sus clientes actuales o buscar nuevos clientes que permitieran incrementar los volúmenes de venta?

\section{La empresa}

Cuidados Veterinarios es una empresa de índole familiar que inició sus operaciones en el 1992 gracias a la visión de Arturo Alva, médico veterinario especializado en los principales sectores pecuarios del país, quien observó que la oferta de servicios veterinarios se concentraba en el rubro de animales domésticos, principalmente, des-

1. De acuerdo con el directorio de las principales empresas y entidades del Perú: [www.creditosperu.com.pe]. atendiendo a las unidades «industriales» del mercado pecuario del país. Es decir, no se atendía adecuadamente un mercado de potenciales compradores con capacidad económica y muy interesados en incrementar la productividad de sus unidades productivas.

El sector pecuario en el Perú se compone, principalmente, de las siguientes especies: aves, vacunos, porcinos, ovinos, caprinos, llamas y alpacas, de las cuales las más importantes son las cuatro primeras.

El doctor Alva creó Cuidados Veterinarios teniendo como objetivo fundamental la importación y comercialización de productos veterinarios dirigidos al sector pecuario del país. Para lograr posicionarse en este mercado, la empresa decidió importar productos de laboratorios de reconocido prestigio mundial, de modo que sus proveedores principales terminaron siendo el laboratorio Zootz Remss y otras compañías del grupo Rhone Poulenc.

A fines de 1996, dos empresas líderes en el mercado mundial de productos veterinarios: Zootz Remss, del grupo Zootz Food, y Brock E.B.L.C., del grupo Brock Herd \& Gold, decidieron fusionarse para constituir Tasmil, convertida en la más importante empresa en el mundo de fabricación e investigación de productos veterinarios y genética avícola.

Este hecho tuvo un impacto directo en la empresa, pues Tasmil decidió que Cuidados Veterinarios sería el distribuidor exclusivo de todas sus líneas en el Perú. Para la empresa del doctor Alva, fue un salto importante, pues tuvo que absorber todos los inventarios de Tasmil existentes en el territorio peruano y encargarse de 
maximizar las operaciones de su representada.

Cuidados Veterinarios ha estructurado sus operaciones en función de tres unidades de negocios claramente definidas: unidad avícola, unidad ganadera y unidad mascotas. La empresa decidió ingresar a atender estos sectores debido al know how desarrollado por sus principales socios, quienes tenían amplia experiencia en estos campos.

La compañía cuenta con 53 trabajadores, de los cuales 21 componen una fuerza de ventas altamente calificada: más de la mitad de los vendedores son médicos veterinarios especializados en el rubro que atienden.

\section{Los sectores atendidos y sus características}

\subsection{Sector avícola}

De acuerdo con el portal agrario del Ministerio de Agricultura (Minag) del Perú (www.portalagrario.gob.pe), la avicultura constituye la actividad más importante del subsector agropecuario, pues representa más del $50 \%$ del PBI pecuario, el $20 \%$ del PBI agropecuario y el 1,8\% del PBI nacional. Asimismo, este sector genera un impacto significativo en otras actividades agrícolas conexas, que también tienen cierta importancia económica para el país. El desarrollo de la cadena de abastecimiento del maíz amarillo duro es un ejemplo de ello.

Si bien hay producción avícola en las tres regiones naturales del país (costa, sierra y selva), algo más del $80 \%$ de la población de aves se concentra en la costa, principalmente en las ciudades más grandes: Lima -la capital-, La Libertad y Arequipa (ver cuadro 1). Es importante señalar que mientras en la costa las unidades avícolas utilizan procesos intensivos de producción, en las otras regiones predomina la crianza artesanal.

La costa peruana cuenta con condiciones idóneas para el desarrollo avícola: poca variación climática durante las diferentes estaciones del año, escasas precipitaciones y una temperatura estable (entre $13{ }^{\circ} \mathrm{C}$ y $29^{\circ} \mathrm{C}$ ). Asimismo, conviene tener en cuenta la infraestructura disponible y la facilidad de acceso en la costa peruana, factores clave de los cuales carecen las otras regiones y que elevan considerablemente los precios de sus productos ${ }^{2}$.

Se puede decir que el peruano prefiere el pollo. Del total de carne consumida en el país (48,8kg/hab), aproximadamente la mitad es de ave, seguida del pescado. Este hecho se explica, por un lado, por el bajo precio comparativo de la carne de ave con respecto a las otras alternativas de consumo y, por otro lado, por los esfuerzos de una industria muy organizada (sobre todo en la zona costera) y con visión de largo plazo. Contar con un mercado interno fuerte le permite al sector potenciar sus actividades; en el Perú, el consumo per cápita de ave es uno de los más altos de la región (ver cuadro 2).

El sector avícola es un oligopolio compuesto por empresas lo suficientemente grandes como para estar consideradas entre las más importantes del Perú. Las prin-

2. De acuerdo con la Asociación Peruana de Profesionales en Logística (Aprolog), el Perú enfrenta un sobrecosto logístico promedio del $30 \%$ por falta de infraestructura básica. 


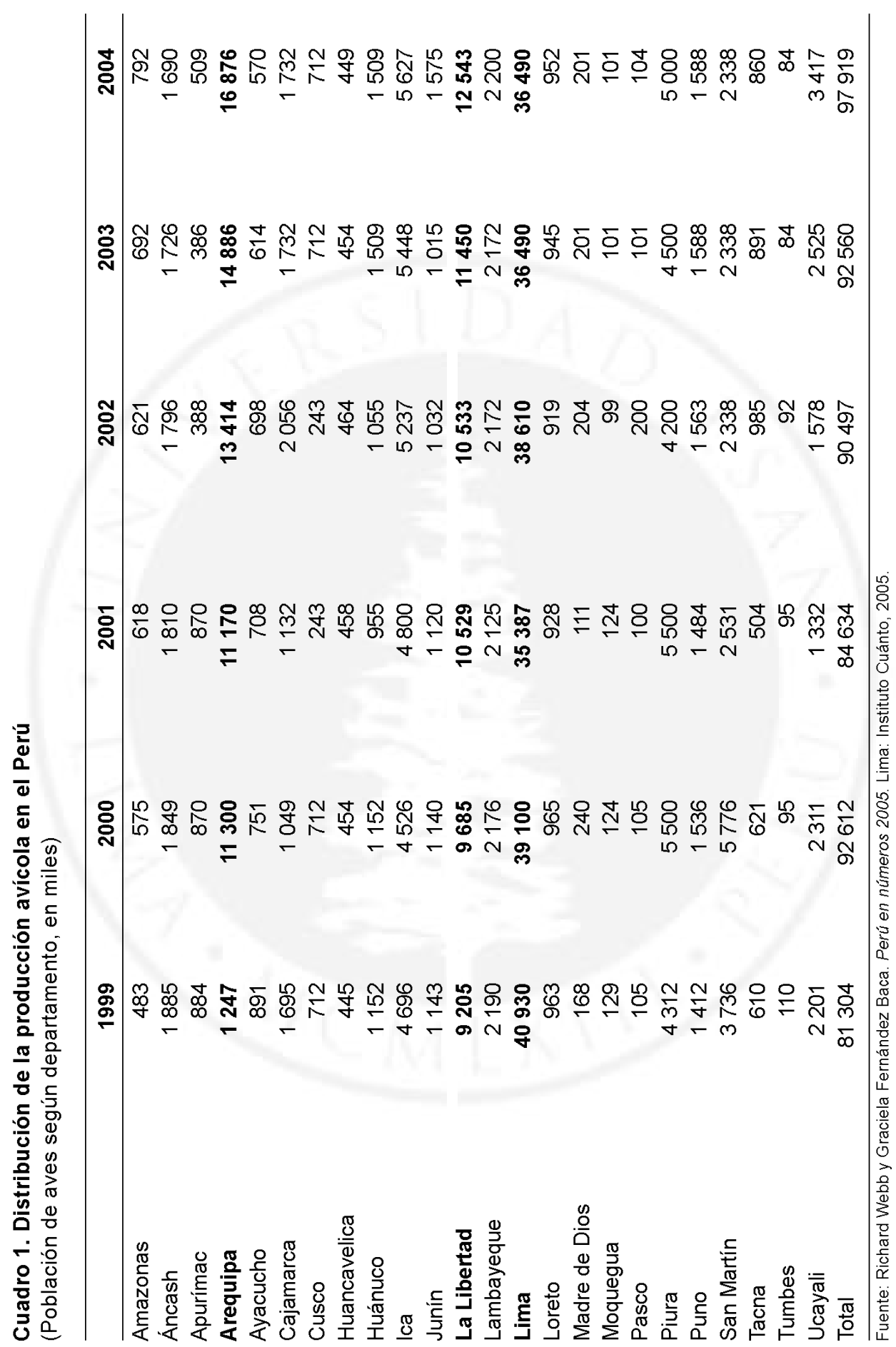




\begin{tabular}{lcc}
$\begin{array}{l}\text { Cuadro 2. Consumo de carnes } \\
\text { en el Perú }\end{array}$ \\
\hline $\begin{array}{c}\text { Tipo } \\
\text { de carne }\end{array}$ & $\begin{array}{c}\text { Consumo } \\
\text { per cápita } \\
\text { (kg/habitante) }\end{array}$ & Participación \\
\hline Ave & 24,2 & $49,59 \%$ \\
Pescado & 14,1 & $28,89 \%$ \\
Vacuno & 5,4 & $11,07 \%$ \\
Ovino & 1,2 & $2,46 \%$ \\
Porcino & 3,6 & $7,38 \%$ \\
Caprino & 0,3 & $0,61 \%$ \\
\hline
\end{tabular}

Fuente: Ministerio de Agricultura (Minag), 2005.

cipales son Corral, Cabaña, Pollos S.A., El Gallo y Germinal. Estas compañías disponen de una elevada capacidad financiera, cuentan con cuadros gerenciales y personal profesional y se mantienen en la búsqueda constante de mejorar la productividad de su gestión.

Actualmente, los principales actores del sector se encuentran organizados en la Asociación Peruana de Avicultura (Apavic) y cuentan con el apoyo del gobierno central para potenciar su desarrollo. Cabe men- cionar que durante los últimos diez años, la producción de pollo se ha duplicado.

El Perú es uno de los principales productores de carne en Sudamérica, sólo superado por Brasil, México y Argentina. Sin embargo, la principal debilidad de la producción radica en sus elevados costos en comparación con los de Brasil o Estados Unidos, inconveniente que podría convertirse en una fuerte amenaza si la construcción de la carretera Interoceánica y la firma del TLC (tratado de libre comercio con Estados Unidos, primer productor mundial) facilita el ingreso de competidores del exterior, por la reducción de los sobrecostos logísticos y las barreras arancelarias, respectivamente.

Otro aspecto que debe considerarse es la evolución de la gripe aviar en el mundo. Si bien en el corto plazo esta epidemia posibilita el acceso a nuevos mercados internacionales a los distintos productos de la industria avícola, en el mediano plazo no se descarta la llegada de la gripe aviar a Latinoamérica, lo cual podría afectar significativamente el desempeño del sector.

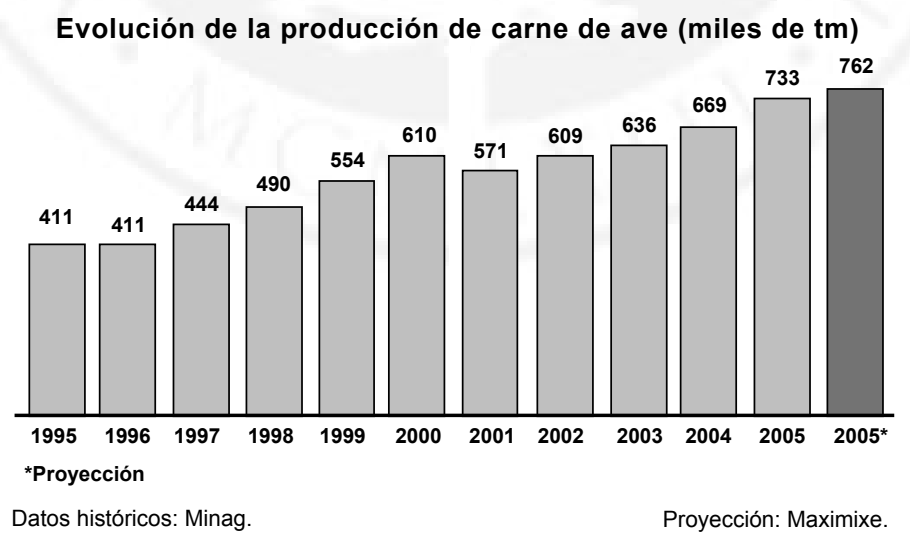

Evolución de la producción de carne de ave 
Cuadro 3. Productividad en la gestión avícola

\begin{tabular}{cc|cc}
\hline $\begin{array}{c}\text { Mejoramiento en el ritmo de crecimiento } \\
\text { de pollos de carne } \mathbf{( 2} \mathbf{~ k g})\end{array}$ & \multicolumn{2}{c}{$\begin{array}{c}\text { Rendimiento en la producción } \\
\text { de carne de pollo (kg/animal) en 2004 }\end{array}$} \\
\hline 1955 & 112 días / 46 semanas & Argentina & 2,20 \\
1960 & 70 días / 10 semanas & Japón & 1,96 \\
1970 & 57 días / 8,2 semanas & Guatemala & 1,90 \\
1985 & 49 días / 7 semanas & Perú & $\mathbf{1 , 9 0}$ \\
1990 & 44 días / 6,2 semanas & Venezuela & 1,88 \\
1999 & 38 días / 5,5 semanas & México & 1,84 \\
\hline
\end{tabular}

Fuente: Minag, Portal Agrario; Maximixe, Análisis sectorial del sector avícola, ago. del 2005.

\subsection{Sector ganadero-vacuno}

Para describir este sector es conveniente subdividirlo en las dos actividades que comprende: la producción de carne y la producción de leche.

\section{Sector ganadero dedicado a la producción de carne}

El sector ganadero dedicado a la producción de carne representó el 8,4\% del valor bruto de la producción agropecuaria peruana (VAB) en el $2004^{3}$. La actividad ganadera se desarrolla, principalmente, en zonas rurales, en unidades ganaderas pequeñas, dispersas, que actúan de manera independiente y con escaso acceso a la tecnología.

Dado lo alejado de las unidades ganaderas, la cadena de comercialización es amplia. Los acopiadores compran el ganado en pie y lo envían, a través de transportistas, a las zonas urbanas (principalmente Lima, la ciudad capital), donde es

3. Riesgos de Mercado Caser (Maximixe). Lima, oct. del 2005. Carne de vacuno. sacrificado (algunas reses ingresan previamente a centros de engorde). La carne fresca se distribuye en el mercado interno, generalmente, a través de carnicerías pequeñas. Toda esta cadena encarece el precio final al consumidor, lo cual explica su bajo consumo per cápita.

En los últimos 15 años, la evolución del rendimiento de la carne de vacuno, en lugar de mostrar un crecimiento, ha experimentado una tasa promedio de decrecimiento de $1,22 \%$, lo cual es indicador de la fragilidad del sector ${ }^{4}$. El desarrollo favorable de la ganadería intensiva pasa por el apoyo del Estado, que ahora no recibe.

Un aspecto que debe mencionarse es que del total de la saca nacional, el 91,36\% sigue la cadena productiva y de comercialización señalada, mientras que el 8,64\% responde a un esquema de engorde intensivo, que consiste en el uso de alimentos concentrados con la finalidad de incremen-

4. Manuel Efraín Rosemberg Barrón. Perspectivas de la ganadería vacuna en el Perú. Lima: Ministerio de Agricultura, 2005. 
Cuadro 4. Producción de carne de vacuno por región (en t)

\begin{tabular}{lrrr}
\hline & $\mathbf{2 0 0 3}$ & $\mathbf{2 0 0 4}$ & $\mathbf{2 0 0 5}$ \\
\hline Cajamarca & 16965,70 & 17878,50 & 20836,00 \\
Lima & 18882,40 & 18389,90 & 19359,20 \\
Puno & 17200,60 & 17186,00 & 17229,90 \\
Cusco & 8600,50 & 11006,50 & 10311,80 \\
Piura & 6806,00 & 9628,00 & 10238,00 \\
Arequipa & 9284,30 & 9211,10 & 9732,60 \\
Ayacucho & 8524,10 & 8566,70 & 9405,00 \\
Huánuco & 8040,90 & 8953,30 & 9010,00 \\
Áncash & 6009,90 & 6186,20 & 6599,00 \\
Lambayeque & 7909,70 & 2794,30 & 2939,20 \\
Otros & 36705,50 & 36561,30 & 37447,60 \\
Total & 144929,60 & 146361,80 & 153108,30 \\
\hline
\end{tabular}

Fuente: Maximixe, feb. del 2006.

tar el peso de los terneros en un breve periodo (3 meses aproximadamente). Por lo general, este sistema lo emplean unidades ganaderas muy pequeñas, que buscan maximizar la relación costo-beneficio en el corto plazo. Por ello estos ganaderos no suelen invertir en productos veterinarios, pues el ganado permanece muy poco tiempo en sus manos.

Finalmente, es necesario mencionar que el Perú negocia actualmente un acuerdo de complementación económica con el Mercosur, lo que podría facilitar el ingreso de carnes procedentes de Argentina y Brasil, principales proveedores de carne del país y cuyos niveles de productividad son elevados.

\section{Sector ganadero dedicado a la producción de leche}

El sector ganadero dedicado a la producción de leche representó el 4,1\% de la producción agropecuaria peruana (VAB) en el año $2004^{5}$. La actividad ganadera para la producción de leche se concentra en tres zonas claramente definidas.

- La cuenca norte, compuesta por los departamentos de Cajamarca, La Libertad y Lambayeque, que concentra el $25 \%$ de la producción.

- La cuenca centro, compuesta por los departamentos de Lima e Ica, que concentra el $17 \%$ de la producción.

- La cuenca sur, compuesta por los departamentos de Arequipa, Moquegua y Tacna, que concentra el $26 \%$ de la producción 6 .

Si bien el Perú cuenta con condiciones favorables para este tipo de ganadería, las unidades ganaderas son muy pequeñas y también dispersas. Por ejemplo, el promedio de la cuenca centro es de 195 vacas por unidad ganadera ${ }^{7}$, lo cual mul-

5. Ibíd.

6. Ibíd

7. Agroenfoque. Lima. Nov., 2005, pág. 60. 
Cuadro 5. Producción nacional de leche de vacuno según departamento (miles de t)

\begin{tabular}{|c|c|c|c|c|c|c|}
\hline & 1999 & 2000 & 2001 & 2002 & 2003 & 2004 \\
\hline Amazonas & 20,30 & 20,60 & 32,30 & 47,80 & 51,30 & 53,40 \\
\hline Áncash & 18,00 & 18,00 & 16,30 & 17,10 & 17,80 & 17,70 \\
\hline Apurímac & 12,60 & 13,00 & 13,60 & 13,80 & 13,00 & 13,20 \\
\hline Arequipa & 220,60 & 245,30 & 255,30 & 264,60 & 272,20 & 286,70 \\
\hline Ayacucho & 31,40 & 32,10 & 16,60 & 28,40 & 19,20 & 16,70 \\
\hline Cajamarca & 146,00 & 153,60 & 178,60 & 203,10 & 208,60 & 208,60 \\
\hline Cusco & 6,40 & 7,80 & 6,30 & 8,60 & 7,90 & 8,70 \\
\hline Huancavelica & 17,90 & 17,90 & 17,80 & 17,70 & 17,60 & 17,60 \\
\hline Huánuco & 10,90 & 11,30 & 14,90 & 15,10 & 15,40 & 18,00 \\
\hline Ica & 17,60 & 15,00 & 15,00 & 15,00 & 16,00 & 16,50 \\
\hline Junín & 18,00 & 18,00 & 18,10 & 18,30 & 18,30 & 18,40 \\
\hline La Libertad & 48,40 & 55,10 & 57,00 & 65,40 & 70,80 & 75,60 \\
\hline Lambayeque & 25,20 & 27,00 & 27,80 & 28,20 & 28,40 & 28,00 \\
\hline Lima & 134,00 & 153,80 & 179,70 & 184,90 & 191,80 & 199,80 \\
\hline Loreto & 0,60 & 0,50 & 0,50 & 0,40 & 0,60 & 0,70 \\
\hline Madre de Dios & 2,00 & 1,90 & 1,10 & 1,80 & 1,60 & 1,70 \\
\hline Moquegua & 14,80 & 15,10 & 15,30 & 15,00 & 15,80 & 16,10 \\
\hline Pasco & 12,50 & 12,70 & 16,50 & 16,80 & 15,90 & 16,20 \\
\hline Piura & 32,20 & 37,00 & 29,30 & 36,00 & 37,90 & 27,30 \\
\hline Puno & 19,00 & 19,70 & 24,20 & 25,20 & 32,70 & 38,60 \\
\hline San Martín & 5,00 & 5,30 & 6,40 & 6,20 & 7,70 & 11,20 \\
\hline Tacna & 26,50 & 25,50 & 25,50 & 25,40 & 25,40 & 25,60 \\
\hline Tumbes & 0,30 & 0,30 & 0,30 & 0,30 & 0,50 & 0,40 \\
\hline Ucayali & 0,40 & 0,60 & 1,40 & 1,40 & 2,10 & 2,60 \\
\hline Autoconsumo & 172,80 & 160,20 & 145,40 & 137,40 & 137,50 & 145,50 \\
\hline Total & 1013,40 & 1067,30 & 1115,20 & 1194,20 & 1226,00 & 1264,80 \\
\hline
\end{tabular}

Fuente: Richard Webb y Graciela Fernández Baca. Perú en números 2005. Lima: Instituto Cuánto, 2005. 
tiplica los costos de distribución de los productos de Cuidados Veterinarios.

Es importante mencionar que en el Perú el consumo per cápita de leche es de 56 litros por habitante al año, muy por debajo del promedio de la región y de lo recomendado por la FAO (120 litros por habitante al año). Si bien la evolución del consumo per cápita muestra un estancamiento, a mediano plazo se puede detectar una oportunidad de desarrollo para el sector, dada su relación directa con el consumo, pues se estima que el PBI per cápi$\mathrm{ta}^{8}$ se incrementará.

La producción de leche es absorbida por la gran industria y por la agroindustria rural, las que adquieren cerca del $90 \%$ de la producción local. Es necesario des- tacar que los principales clientes son empresas grandes, como Gloria S.A., Nestlé y Laive, que realizan el acopio de leche en las propias unidades ganaderas y son, en parte, responsables de la evolución favorable de la producción lechera en el país. Sin embargo, normalmente estas empresas no invierten en la adquisición de ganado, más bien se concentran básicamente en la compra de leche.

\subsection{El sector de mascotas}

El negocio de productos para mascotas está creciendo rápidamente en todo el mundo. Por ejemplo, actualmente se estima que el mercado mundial de alimentos balanceados para perros y gatos asciende a 35 mil millones de dólares, de los cuales el 5\% corresponde a América Latina9 ${ }^{9}$.

\section{Cuadro 6. Comida para gatos y perros: multimix de consumo}

Perfil: Comida para gatos-comida para perros

Cuadro: Según lugar de compra

Ciudad: Lima Metropolitana

\begin{tabular}{l|rrrr|rrrr}
\hline & \multicolumn{5}{|c|}{ Comida para gatos } & \multicolumn{4}{c}{ Comida para perros } \\
\hline & Total & A/B & N.S.E. & D $/ E$ & Total & A/B & C & D/E \\
\hline \% consumidores & & & & & & & \\
del producto & $100,00 \%$ & $100,00 \%$ & $100,00 \%$ & $100,00 \%$ & $100,00 \%$ & $100,00 \%$ & $100,00 \%$ & $100,00 \%$ \\
Autoservicio & $50,10 \%$ & $71,40 \%$ & $64,40 \%$ & $0,00 \%$ & $42,40 \%$ & $53,10 \%$ & $29,40 \%$ & $6,40 \%$ \\
Puesto mercado & $30,10 \%$ & $19,50 \%$ & $8,50 \%$ & $72,30 \%$ & $23,60 \%$ & $18,20 \%$ & $24,70 \%$ & $57,00 \%$ \\
Bodega & $16,40 \%$ & $7,10 \%$ & $27,00 \%$ & $18,20 \%$ & $18,60 \%$ & $15,80 \%$ & $26,20 \%$ & $15,90 \%$ \\
Ambulante & $2,60 \%$ & $0,00 \%$ & $0,00 \%$ & $9,50 \%$ & $13,90 \%$ & $12,90 \%$ & $13,80 \%$ & $20,60 \%$ \\
Otro lugar & $0,80 \%$ & $2,00 \%$ & $0,00 \%$ & $0,00 \%$ & $1,50 \%$ & $0,00 \%$ & $5,80 \%$ & $0,00 \%$ \\
\hline
\end{tabular}

Fuente: Compañía de Investigación de Mercado y Opinión Pública.

8. De acuerdo con el Marco Macroeconómico Multianual 2006-2008 (Ministerio de Economía y Finanzas), se espera que el PBI per cápita crezca de manera paulatina desde 2484 dólares en el 2005 a 3898 dólares en el 2015.
9. D. Mayorga, P. Araujo y M. Schwalb. Las mejores prácticas del márketing: casos ganadores de los Premios Effie 2004 (Caso Mimaskot). Lima: Universidad del Pacífico, 2005. 
El Perú no es ajeno a esta tendencia: en los últimos años se observa un cambio de actitud en el cuidado de estas mascotas, lo que repercute en el crecimiento del sector. Sin embargo, si bien se da un incremento, todavía no se observa un crecimiento explosivo.

El cambio más notorio en cuanto al cuidado de las mascotas se refleja en la alimentación. Hasta hace algunos años, perros y gatos comían productos elaborados de manera doméstica: abundantes carbohidratos y algunos elementos cárnicos. El consumo per cápita de alimentos para perros, por ejemplo, ascendía a 0,4 kilos anuales, consumo menor al de otros países, como Venezuela $(1,4 \mathrm{~kg})$, Colombia $(1,6 \mathrm{~kg}) \mathrm{y}$ Chile (4,5 kg). Sin embargo, esta situación va cambiando: el crecimiento de esta categoría en el año 2003 ha superado en $18 \%$ respecto al 2002, y en el primer trimestre del 2004 ha sido de $38 \%$.

En este mercado podemos distinguir el mercado de consumo y el mercado especializado. El primero comercializa productos de uso cotidiano de las mascotas, como alimentos balanceados y accesorios, y se compone de pet shops, autoservicios, bodegas, puestos de mercados y ambulantes. Cabe señalar que los autoservicios y los puestos de mercado representan los canales de distribución más importantes en este segmento.

Por su parte, el mercado especializado lo forman agroveterinarias, veterinarias y también pet shops. Cuidados Veterinarios visita estos locales, básicamente, para atender consultas de salud sobre las mascotas y para realizar acciones preventivas. Según datos de la Sunat, en Lima funcionan 18 pet shops, y en todo el país hay 173 veterinarias registradas.
Los productos médicos para la atención de mascotas se distribuyen a través de estos canales. Sin embargo, estos locales distribuyen productos como alimentos, accesorios, antiparasitarios, etcétera, en forma complementaria a su actividad principal; por tanto, no representan un canal de salida importante para las líneas complementarias.

Finalmente, es importante señalar que se espera una evolución favorable en este mercado, porque se encuentra en una etapa inicial de su desarrollo y porque está íntimamente ligado a la evolución del PBI per cápita, vale decir, a los niveles de ingreso de la población. Como se mencionó anteriormente, se espera un crecimiento importante de este indicador en los próximos años.

\section{Cartera de productos de la empresa}

La empresa concentra sus operaciones en tres unidades estratégicas, tal como se puede observar a continuación.

\section{Cuadro 7. Productos Veterinarios:} unidades de negocios

\begin{tabular}{cc}
\hline $\begin{array}{c}\text { Unidad estratégica } \\
\text { de negocio }\end{array}$ & $\begin{array}{c}\text { \% de participación en } \\
\text { los ingresos de } \\
\text { la empresa }\end{array}$ \\
\hline Avícola & $80 \%$ \\
Mascotas & $17 \%$ \\
Vacuno & $3 \%$ \\
\hline
\end{tabular}

La unidad estratégica de negocio más importante de la empresa es la avícola, no sólo porque representa el $80 \%$ de sus ventas, sino porque además concentra sus operaciones en unos cuantos clientes de gran poder económico, con capacidad de 
compra por volumen (dada la naturaleza de sus operaciones) y ubicados en zonas de fácil acceso.

\subsection{Unidad estratégica de negocio subsector avícola}

Esta unidad de negocio se desarrolla en función de tres líneas de productos: la línea de productos biológicos (vacunas), la línea de productos nutricionales (complementos vitamínicos) y la línea de medicamentos.

Los principales competidores en esta unidad estratégica son Folks, Mundial y Chicken Perú. Folks Perú es una empresa subsidiaria de Folks Internacional, empresa que facturó más de 1024 millones de euros en el 2004. Folks Perú es una empresa sumamente dinámica que participa en los mercados avícola, ganadero y de animales de compañía. Para los dos primeros sectores cuenta con doce representantes de ventas; y para el segundo, con tres distribuidores exclusivos.

Mundial es una empresa de capitales nacionales que no solamente cubre el rubro de salud animal, sino que se dirige a otros segmentos: ingredientes para alimentos, productos para la nutrición y salud animal, e ingredientes naturales. Los productos de la división de nutrición y salud animal se producen localmente, lo que otorga a la empresa una ventaja significativa de precios. Mundial cuenta con 250 trabajadores y factura anualmente 50 millones de dólares.

Chicken es una empresa multinacional que opera en Bolivia, Brasil, Chile, Colombia, Guatemala, Perú, Venezuela y Estados Unidos. Cuenta con tres divisiones, de las cuales sólo una está enfocada a la salud animal. Si bien las importaciones de la empresa son significativas en la industria, los volúmenes de importación se encuentran estancados.

\section{La línea de productos biológicos}

Los productos biológicos constituyen la principal línea de Cuidados Veterinarios; representa el $80 \%$ de sus ventas en este sector. Consiste, básicamente, en vacunas para el cuidado de las aves, productos considerados «indispensables» por las granjas, ya que evitan que los animales mueran por causa de virus o de enfermedades comunes. Es una línea de productos básicamente preventiva.

La vida de los pollos en granja es de 40 días aproximadamente. Durante este periodo el animal tiene que ser vacunado tres veces. En esta línea, Cuidados Veterinarios es la líder del mercado, pues su participación es superior al 50\%. Quizá la razón más importante de este desempeño es que la empresa mantiene la representación exclusiva de los productos Tasmil $^{10}$ en el Perú.

Esta línea, la tradicional de la empresa, se compone de productos de alto valor percibido y elevado margen de contribución unitario. Sin embargo, se trata de un mercado maduro para Cuidados Veterinarios, con escasas proyecciones de crecimiento, tanto por la penetración de este tipo de producto en la industria como por su alta participación en el mercado.

10. Tasmil es la compañía líder mundial en salud animal. En el sector avícola maneja una amplia cartera de vacunas y sistemas de administración de vacunas para proteger a las aves en las distintas etapas de su vida en granja. 


\section{La línea de productos nutricionales}

La línea de productos nutricionales es relativamente nueva para la empresa, pero es de uso permanente en la industria, pues los productos no sólo se usan como complemento alimenticio para las aves, sino también como antibióticos que ayudan a prevenir la aparición de ciertas enfermedades. Al tener como parte de sus componentes antibióticos, es necesario que en esta línea haya un cambio de componentes cada cierto tiempo.

Esta línea representa cerca del 15\% de las ventas de Cuidados Veterinarios en esta categoría, pero su participación en el mercado es incipiente: apenas llega al $2 \%$.

Para esta línea de productos, Cuidados Veterinarios viene trabajando desde el 2000, bajo representación exclusiva, con Cronin, empresa considerada entre las principales del mundo en el rubro de nutrición para animales. Las importaciones de Cuidados Veterinarios en esta línea de productos han crecido de manera exponencial; entre el 2005 y el 2004 aumentaron en alrededor de $300 \%{ }^{11}$.

Esta línea de negocio se caracteriza por altos volúmenes de compra a lo largo del tiempo, de manera continua, pero los márgenes que se manejan son pequeños.

\section{La línea de productos médico- veterinarios}

Este es un producto de carácter reactivo principalmente, pues la compra de este tipo de productos se realiza de manera esporádica, cuando ha aparecido alguna enfermedad en los animales. Es un mer-

11. Fuente: Aduanet. cado pequeño, estático y con muchos proveedores. Representa el 5\% de las ventas de Cuidados Veterinarios en la categoría avícola.

\subsection{Unidad estratégica de negocio mascotas}

El mercado de mascotas experimenta un franco crecimiento, posee márgenes interesantes y casi no está siendo explotado. Está segmentado en dos grupos claramente diferenciados: el de consumo y el especializado y es atendido a través de cuatro líneas de productos: alimentos, antiparasitarios, accesorios y vacunas.

\section{La línea de alimentos}

Esta es la línea más importante del mercado y la que muestra un mayor crecimiento. La mayoría de los productos se dirigen al segmento de consumo a través de supermercados, mercados y bodegas.

Puesto que la empresa acostumbra trabajar bajo el esquema de distribución exclusiva, obtuvo la representación de Dietetic Enterprise de Lorn's, empresa líder en el sector de alimentos del ámbito mundial. Si bien se trata de productos de alto valor percibido, una limitación de Cuidados Veterinarios es que, por directiva de la casa matriz, no se pueden vender a través del mercado de consumo, sino por intermedio de los canales que expenden productos especializados: pet shops, veterinarias y agroveterinarias.

El impacto de esta restricción se nota en la escasa participación de la empresa en los mercados de productos representados. En esta línea de productos compite con empresas locales e internacionales. Entre las empresas locales se ha podido 


\section{Cuadro 8. Multimix de consumo de comidas para perros}

Perfil: Comida para perros

Cuadro: Penetración-según marcas

Ciudad: Lima Metropolitana

\begin{tabular}{|c|c|c|c|c|}
\hline & \multirow{3}{*}{ Total } & \multicolumn{3}{|c|}{ Comida para perros } \\
\hline & & \multicolumn{3}{|c|}{ Nivel socioeconómico } \\
\hline & & $A / B$ & C & $\mathrm{D} / \mathrm{E}$ \\
\hline$\%$ consumidores del producto & $100,00 \%$ & $31,60 \%$ & $20,60 \%$ & $12,50 \%$ \\
\hline Mimaskot & $8,30 \%$ & $10,10 \%$ & $11,90 \%$ & $5,80 \%$ \\
\hline Granel & $4,20 \%$ & $5,00 \%$ & $4,40 \%$ & $3,80 \%$ \\
\hline Ricocan & $3,80 \%$ & $9,60 \%$ & $3,10 \%$ & $2,10 \%$ \\
\hline Pedigree & $2,30 \%$ & $5,00 \%$ & $2,60 \%$ & $1,30 \%$ \\
\hline Alpo & $2,00 \%$ & $7,00 \%$ & $1,40 \%$ & $0,40 \%$ \\
\hline Babycan & $0,90 \%$ & $2,10 \%$ & $1,00 \%$ & $0,40 \%$ \\
\hline Supercan & $0,80 \%$ & $2,60 \%$ & $1,00 \%$ & $0,00 \%$ \\
\hline Proplan & $0,60 \%$ & $2,20 \%$ & $0,60 \%$ & $0,00 \%$ \\
\hline Dog Chow & $0,20 \%$ & $1,10 \%$ & $0,00 \%$ & $0,00 \%$ \\
\hline Hill's Sport & $0,10 \%$ & $0,60 \%$ & $0,00 \%$ & $0,00 \%$ \\
\hline Doguitos & $0,10 \%$ & $0,60 \%$ & $0,00 \%$ & $0,00 \%$ \\
\hline
\end{tabular}

Fuente: Compañía de Investigación de Mercado y Opinión Pública.

identificar a las siguientes fábricas: Comestibles S.A., Yupura Perú, S.A., Molinos Sonai S.A., Molino Taurus, S.A. y Paisaje S.A. La actividad principal de estas empresas es la producción de alimentos para ganado, aves y animales domésticos. Asimismo, hay empresas importadoras de marcas extranjeras de alta aceptación en el mercado local.

\section{La línea de antiparasitarios}

El producto estrella de la empresa en esta categoría es Quick. Hasta hace algunos años, Cuidados Veterinarios, como representante exclusivo de Tasmil, era no sólo el único proveedor de este producto en el mercado, sino que, además, no tenía competidores, pues se trataba de un producto protegido por patentes ( 7 años). Esta si- tuación le permitía obtener márgenes amplios.

Sin embargo, el periodo de protección de la patente caducó y empezaron a ingresar nuevos competidores que atacaron el mercado con una estrategia audaz de precios. Los márgenes de esta línea de producto cayeron mucho y la participación de Quick en el mercado viene cayendo año tras año.

\section{La línea de accesorios}

El mercado para esta línea de negocio es pequeño; si bien es una plaza con escasas barreras de entrada, permite economías de escala en compras por volumen. Manejar compras de contenedores completos abarata el aprovisionamiento de productos y 
genera el margen suficiente para obtener rentabilidad. En este mercado, Cuidados Veterinarios participa en ambos segmentos: el de consumo y el especializado.

\section{La línea de vacunas}

Esta es una línea donde la competencia no ha permitido el ingreso de Cuidados Veterinarios. Chong, el principal competidor, maneja una política de precios muy atractiva y unas condiciones de crédito con las cuales Cuidados Veterinarios no puede operar.

\subsection{Unidad estratégica de negocio ganado vacuno}

Con relación a esta unidad de negocio, Cuidados Veterinarios utiliza, al igual que en su línea avícola, la representación exclusiva de Tasmil. Sin embargo, como se ha mencionado, las características de este sector pecuario son muy diferentes a las del sector avícola. Como Tasmil es una empresa transnacional, sus dosis no necesariamente se adaptan al mercado peruano. Específicamente, se debe señalar que las dosis de Tasmil resultan demasiado grandes para el mercado local, pues están dirigidas a unidades ganaderas de mayor tamaño que las peruanas.

Para atender el mercado peruano, han aparecido una serie de empresas maquiladoras que adquieren el producto y lo reenvasan de tal manera que se adecúan a la realidad del mercado peruano ganade- ro. Sin embargo, esta opción no es rentable por el escaso margen obtenido. Por otro lado, las restricciones impuestas por Tasmil a Cuidados Veterinarios no hacen posible el uso de esta opción, ni la empresa está diseñada para actividades de maquila.

\section{El razonamiento del señor Alva}

La empresa está altamente concentrada en un sector industrial, específicamente en una línea de producto. La característica principal de venta es la representación exclusiva de la marca Tasmil. Por otro lado, el mercado avícola es un oligopolio donde operan pocas empresas con altos volúmenes de compra. Dado el tamaño del mercado y la concentración de la demanda, no sería descabellado que Tasmil pudiera ingresar a atender directamente este sector pecuario o retirar la representación exclusiva que le otorgó a Cuidados Veterinarios.

Por todo ello, el señor Alva cree que es necesario hacer algo para disminuir su riesgo. Actualmente considera líneas paralelas de negocios, pues cree poco probable que la empresa siga creciendo a través de sus líneas tradicionales de productos biológicos para el sector avícola, pero debe ser muy cuidadoso en la elección, ya que la escasa liquidez de Cuidados Veterinarios y sus limitados recursos financieros la obligan a priorizar sus actividades de gestión e inversión. 